\title{
A CASE OF COMPLETE ABSENCE OF THE VISUAL SYSTEM IN AN ADULT.
}

\author{
BY WILLIAM G. SPILLER, M.D.
}

Assistant Clinical Professor of Nervous Diseases and Assistant Professor of Neuropathology in the University of Pennsylvania; Clinical Professor of Nervous Diseases in the Women's Medical College of Pennsylvania.

(Read before the College of Physicians of Philadelphia, November 6, 1901).

COMPLETE absence of the visual system is of very rare occurrence, especially in an adult. The study of the case I report in this paper should be of more value than one of agenesia of the visual system in a newborn child, because in the latter portions of the brain not belonging to the visual system are imperfectly developed, and faulty conclusions may therefore be drawn.

The boy, T. S., according to the case-books of the Pennsylvania Training School for Feeble-minded Children, was born in 1878, and was admitted to the School March 12, 1895. At the time of admission he weighed thirty-eight pounds, and his height was $3 \mathrm{ft} .7$ in. He was an idiot, absolutely helpless, and very unclean. He could say "mamma," and nothing more. He was passionately fond of music. At the time of admission to the school he appeared to be about 8 or 10 years of age.

I saw him on July 30, 1897, and made a few notes on his condition. I regarded the case at that time as one of cerebral spastic paraplegia of the lower limbs, with absence of the eyeballs. 'Ihe boy was unable to stand alone, and if he were stood up and not supported he fell backward. When supported he could take a few steps, but in doing so one lower limb was moved in front of the other (cross-legged progression). His knee-jerks were exaggrerated, but ankle-clonus was not obtained. He could move his lower limbs while he was sitting, and there was not excessive

- From the William Pepper Laboratory of Clinical Medicine (Phebe A. H.carst Foundation).

vol. XXIV. 
rigidity of these limbs on passive motion. He uttered only a few monosyllables. His palpebral fissures were very small. He died March 2, 1900, and the necropsy was made two days later. The necropsical notes I shall use only in so far as they are of importance for the understanding of the case.

The body was that of a child apparently about 12 years of age (see fig. 1), though in reality his age was 22 years. No signs of puberty were present. The testicles were not in the scrotum; the right testicle, quite small, was found in the inguinal canal just above the pubes. The genitalia were like those of a child of 10 years. No hairs were found on the pubes, or in the axillæ, or on the face. The circumference of the head was 183 inches; the length of the body was 47 inches. The right upper limb could not be fully extended at the elbow, while the left upper limb could be fully extended. Slight contracture of the fingers of each hand was observed, so that the fingers could not be fully extended when the hands were fully extended at the wrists. The fingers could be fully extended when the hands were flexed at the wrists, as the contracture of the flexors of the fingers did not then interfere with the extension of the fingers. The lower limbs were slightly flexed at the knees, and could not be fully extended.

The palpebral fissure of each eye was very small, and the orbits contained only a small amount of what appeared to be fibrous connective tissue. Unfortunately, permission had not been given for the removal of the orbital contents. Nothing resembling an eyeball was seen.

The optic foramina did not exist, and it was impossible to find an opening in the usual situation of these foramina, and here there were merely slight depressions in the skull. No trace of optic nerves, chiasm, or optic tracts could be found (see fig. 2). There was no sign of an external geniculate body on either side, and the thalamus on each side had nothing resembling an optic tract passing from it. The posterior part of each thalamus was rounded and larger perhaps than one would expect to find it in a case of complete agenesia of the visual system (see fg. 3.) The brain was small, firm, and not odematous. The left ascending frontal convolution in the centre for the upper limb was very small (see fig. 4), to which fact possibly was due the contracture of the right forearm (see fig. 1), because of imperfect development of the nerve fbres innervating this limb. The anterior colliculi of the corpora quadrigemina were fully as large and as well developed as the posterior. The occipital lobes were small, and 
the cuneus on each side (see fig. 5) was small, and the calcarine fissure short. The lower olives were covered by thick bands of nerve-fibres-i.e., the external arcuate fibres were unusually well developed.

The spinal cord was small, but not otherwise abnormal.

The brain was hardened in formaline, and the basal ganglia of one hemisphere were cut in serial sections, while those of the other hemisphere were preserved intact as a gross specimen. Sections were made through the cortex of the left calcarine fissure, and stained by thionin and by Weigert's hæmatoxylin method. Frontal sections were made through the right occipital lobe in order that the optic radiations might be studied when cut transversely. The nerves supplying the ocular muscles were also cut and examined.

The cortex of the left calcarine fissure stained by thionin contained many nerve-cell bodies, but they were possibly slightly less numerous than are the cell bodies in sections from the corresponding region of a normal brain, especially in the third, fourth, and fifth layers. The giant-cells of the third layer were represented, but were possibly not as numerous as in a normal brain. The fibres of Vicq d'Azyr of the cortex of the calcarine fissure were not very distinct in sections stained by Weigert's hæmatoxylin method, but they were not entirely absent.

The optic radiations in the frontal sections of the occipital lobe were not entirely absent, but the area occupied by them was not very distinct.

Meynert's commissure was normal, and stained well by Weigert's hæmatoxylin method (see fig. 6).

Careful examination of the serial sections failed to show the slightest trace of an external geniculate body on either side, although the internal geniculate body was well developed. Fig. 6, a photograph from one of the sections, shows the internal geniculate body in its normal size, as well as the inferior posterior portion of the optic thalamus, but the external geniculate body, which should be present in a section at this level, is entirely absent. Comparison with fig. 326 of the Anatomie des Centres Nerveux, vol. i., by J. and A. Dejérine, is instructive. This drawing is from a section cut in the same direction as that represented in fig. 6 of my series, and shows the normal size of the external geniculate body and an optic tract in a section from this level. In the section of my series represented in fig. 6 there is no trace of an optic tract.

Some few medullated nerve-fibres are found within the pul- 
vinar in my section, but those in the posterior portion of the tbalamus are much fewer than those in the more anterior portions. The contrast is very striking, and is represented in a drawing of a horizontal section (see fig. 7), which should be compared with a corresponding section from a normal brain, as shown in fig. 321 of the Anatomie des Centres Nerveux, by J. and A. Dejérine.

The habenula in the horizontal sections appears well developed, and the fasciculus retroflexus of Meynert is well stained.

In the photograph the anterior colliculi of the corpora quadrigemina appear smaller than the posterior; but this is merely because the former were further from the camera.

Sections through one-half of the oculomotor nuclei stained with thionin contain many nerve-cell bodies belonging to these nuclei, and these cell bodies stain well with thionin and appear to be normal. They are possibly not as numerous as cell bodies in oculomotor nuclei of a normal brain. Sections through one-half of the oculomotor nuclei were also stained by the Weigert hæmatoxylin method.

Transverse sections of the extracerebral portion of the left oculomotor nerve near the cerebral peduncles stained with ammonium carmine show an apparently normal nerve. The nerve is possibly smaller than an oculomotor nerve from a normal brain, but it contains no sclerotic areas. It contains, however, some areus in which very fine nerve-fibres form distinct groups, but there is no scarcity of large nerve-fibres.

Transverse sections of the right oculomotor nerve are very similar to those of the left oculomotor nerve. The sections stain well by Weigert's hæmatoxylin method.

One of the trochlear nerves in transverse sections, stained either by ammonium carmine or Weigert's hæmatoxylin, appears to be normal, and contains nerve-fibres of good size. The nervefibres stain well with the Weigert hæmatoxylin, but in sections so stained the nerve-fibres are perhaps not quite so close together as in the trochlear nerve from a normal brain.

The nerve-fibres of the left abducent nerve stained in transverse sections by acid fuchsin are exceedingly small, much smaller than those of the oculomotor and trochlear nerves, but they stain well with Weigert's hæmatoxylin.

The right abducent nerve in transverse sections stained by acid fuchsin contains many nerve-fibres that are much largel than any of those found in the left abducent nerve, but it contains also many sclerotic areas. Some of the nerve-bundles stained $b$. 
Weigert's hæmatoxylin method contain very few medullated nerve-fibres. The contrast between the abducent nerves and the other two ocular nerves on each side is most striking (see fig. 8).

The nucleus of each abducent nerve contains many nerve-cell bodies which, with the thionin stain, appear to be normal.

The nerve-cell bodies of the anterior horns of the spinal cord in the cervical and lumbar regions stained with thionin are normal, but possibly a little smaller than those usually present in the anterior horns. No sclerotic areas are found in the lateral columns, neither are the nerve-fibres in these columns unusually fine in transverse sections.

von Monakow gives as primary optic centres the external seniculate body, the surface of the pulvinar, and the superficial gray matter of the anterior colliculi of the corpora quadrigemina. He says that Stilling, Bernheimer, and holliker believed that visual fibres arise also in the subthalamic body, the internal geniculate body, and the tuber cinereum. The anterior colliculi of the corpora quadrigemina in man, according to his view, have a very subordinate rôle in vision, and he believes that about 80 per cent. of the optic nerve-fibres terminate in the external geniculate body. My case of congenital absence of the central visual system confirms these views of von Monakow. It shows also that Meynert's commissure and the habenula are not part of the visual system. According to von Monakow (Gehirnpathologi) centrifugal fibres-i.e., fibres having their origin in the visual cortex-are present in the optic radiations and pass to the inter-brain and mid-brain. The giant pyramidal cell bodies of the third layer in the cortex of the occipital lobe give off axones, which pass by way of the optic radiations and the arm of the anterior colliculus of the corpora quadrigemina, to terminate in the superficial medullary substance and gray matter about the aqueduct of Sylvius in the anterior colliculus. The Vicq d'Azyr fibres, he says, are often in a double row in the occipital lobe. They come, in greater part, from the fibres of the optic radiations which pass to the third layer of the cortex, but some of the Vicq d'Azyr fibres may belong to an association system. In long-standing lesions of the optic radiations the Vicq d'Azyr 
fibres are very atrophic, and the double row of these fibres is scarcely recognisable. In my sections the comparatively few fibres passing to the pulvinar are doubtless the fibres described by von Monakow as arising in the occipital lobe, and in my sections the Vicq d'Azyr fibres are not very distinct.

von Leonowa has examined the microscopical sections from four cases of anophthalmia and three cases of atrophy of the eyeball, but in all these death occurred within a few months after birth. In all her cases the posterior colliculi of the corpora quadrigemina were intact, but the anterior colliculi were affected. von Leonowa ${ }^{1}$ concludes from a study of the cases mentioned above that the posterior colliculi of the corpora quadrigemina have no relation to vision, while the anterior have; that the relation of the external geniculate body to the visual fibres is a very intimate one; and that the subthalamic body has no relation to vision. In none of her cases was the absence of the visual system complete, and all the brains were from children in the early periods of extra-uterine life.

In her cases the nuclei of the third, fourth, and sixth cranial nerves were intact, and this integrity is explained by von Leonowa as a consequence of the existence of ocular muscles. The external ocular muscles may be well developed, even though the eyeballs are exceedingly atrophic, as in a case of anophthalmia in a person of 27 years, reported by Haab, in whom the ocular muscles were normal in size and shape, although the ocular bulbs were very small. The nerves to the ocular muscles need not be absent in congenital defect of the visual system. In my case there was apparently no eyeball on either side, but in the small amount of fibrous tissue at the posterior portion of each orbit muscles may bave existed, even though they bad little or no function. One might suppose that in the case I have reported the third, fourth, and sixth nerves would have been very imperfectly developed in conformity with the law of Gudden, according to which atrophy in a neurone follows injury to this neurone. It should be remembered, however, that in

'Archiv. für Psychiatrie, 1896, vol. xxvii., p. 53. 
my case the nerves supplying the ocular muscles were not injured, and may have been in union with ocular muscles, as in Haab's case.

It has been shown by von Leonowa, ${ }^{1}$ in the report of a nuch-quoted case, that nerve-fibres may develop, although they apparently have no function. She examined a fœtus in which the brain and spinal cord had not developed, and yet the vertebral canal was filled in its entire extent by posterior nerve-roots. Some of the fibres of the posterior roots extended from the lower part of the canal to the upper, as they would have done if they had formed the posterior columns of a normal spinal cord. All the posterior roots ended blindly, and seemed to have sought a spinal cord in which to bury themselves. No anterior roots were found. All the peripheral nerve-fibres present had their origin in the spinal ganglia, and belonged, therefore, to the sensory system. The muscles of the limbs appeared fully normal, although no motor nerves existed. Most of the axis cylinders of the posterior roots were non-medullated or had only a thin medullary sheath. The cell bodies of the spinal ganglia were as numerous and as well developed as in a normal foetus. The absence of medullary sheaths on the posterior roots, von Leonowa thinks may have been caused by the amniotic fluid, inasmuch as medullary sheaths were present on axones that were covered by the skin.

Gade $^{2}$ and Karl and Gustaf Petrén have found posterior roots growing into the spinal canal in complete failure of development of the spinal cord. I am unable to determine from the statements of the latter authors whether in their case or in Gade's the individual fibres of the posterior roots were normal or not.

With the results of these investigations in mind we can understand that nerves might grow equally well into the orbits and persist, even though the ocular muscles were absent. Indeed, von Leonowa and Karl and Gustaf Petrén have shown also that in early life muscles may be developed, although no motor nerves exist, so that the converse may

1 Neurologisches Centralblatt, 1893.

₹ Gadé, cited by Karl and Gustaf Petrén. Virchow's Archiv., 1898, B. 151 
well be true-i.e., motor nerves probably may exist, although the muscles are not developed. All the ocular nerves in my case, however, were not normal. The two sixth nerves were very imperfectly formed.

Meynert's fibres are asserted by some to be the connection between the optic nerves and the oculomotor centres, a.lthough such distinguished cerebral anatomists as Edinger and von Monakow tell us that the anatomical proof for this assertion is wanting. Recent investigations seem to indicate that some of these Meynert fibres (for there are more than one band of fibres named after Meynert) belong to a bundle extending into the anterior columns of the spinal cord. In iny case the Meynert fibres in the corpora quadrigemina were well developed, although there could have been no reflex fibres from the iris to the oculomotor centres, so that we must conclude that the function of these Meynert fibres cannot be merely to serve the pupillary reflex. I am unable to give the course of the so-called pupillary fibres from a study of my preparations, as the fibres in the region of the corpora quadrigemina form an intricate network.

It is not surprising that cell bodies should be numerous in the cortex of the calcarine fissure in a case of congenital absence of the visual system. Some of these cell bodies have axones which are situated in the optic radiations and terminate in the primary optic centres-i.e., the external geniculate body, the pulvinar of the thalamus, and to a less extent the anterior colliculi of the quadrigeminal bodies, and these fibres probably are not directly concerned in vision. The visual cortex is intimately connected with widely separated portions of the brain, as shown both by physiological and anatomical investigations. The movements of the limbs, the functions of speech, and especially the movements of the eyeballs, are in intimate relation with vision, and important association tracts between the visual area and other parts of the brain can be demonstrated anatomically. The cell bodies of the visual cortex must have axones in relation with remote parts of the cerebral cortex, and, therefore, it is not surprising that these association neurones are developed even in a case of congenital defect of the visual 
system. von Monakow represents in his Gehirnpathologie (p. 265) a section of a calcarine cortex from a case of lesion of the optic radiations. The giant pyramidal cell bodies of the third layer of the cortex had entirely disappeared, and the third, fourth, and fifth layers were degenerated in a way they were not in my case. It should be remembered that in von Monakow's case the axones destroyed were those of cell bodies situated in the calcarine cortex, and that atrophy. of these cell bodies probably followed the injury of their axones. This did not occur in my case, and probably the nerve-fibres present in my specimens in the optic radiations arose in the occipital lobe. It is difficult to understand what the function of these fibres can be.

Although the individual described in this paper was an idiot, we should be cautious in attributing his deficient mental development to the absence of his visual system. It would seem probable, however, that the entire brain was in a state of imperfect development.

The spastic paraplegia of the lower limbs deserves mention. The nerve-cell bodies of the anterior horns in the lumbar cord were normal by the thionin stain, except that they were not so large as the cell bodies of this region-usually are, and the lateral columns did not contain sclerotic areas. This is not the first time I have found an apparently normal spinal cord in congenital spastic paraplegia, and I think the explanation is to be found in an imperfect development of the motor neurones. A deficiency from birth in the number of these neurones would probably have the same result as partial destruction of the central wotor tracts, and in the absence of the development of motor neurones there would be no sclerosis, inasmuch as no space existed to be filled by proliferation of the neuroglia. Indeed, sclerosis may be entirely absent if destruction of nerve fibres occurs in early life, because the growing tissue of the spinal cord fills the space left by degenerated fibres, and proliferation of the neuroglia does not occur. I have seen the spinal cord in a case of infantile hemiplegia presenting little or no sclerosis, although the person from whom this cord was removed had lived many years after the 
paralysis had developed. The case to which I refer was in Dejérine's service. In two cases of congenital spastic paraplegia of the lower limbs I believed I was able to detect an unusual fineness of the nerve-fibres of the pyramidal tracts, but I was unable to do so in this case, T. S.

I am indebted to Dr. de Schweinitz for the reference to a case reported by W. S. Little ${ }^{1}$ in 1885 , in which congenital absence of some portions of the visual system was found. The brain was from a child of ten years of age, who had been an inmate of the Pennsylvania Training School for Feeble-minded Children, and the specimen was examined by A. W. Wilmarth. This specimen and mine have therefore come from the same institution. The child had had bi-temporal hemianopsia. There was no optic commissure, but a small projection was found on the inner side of each nerve where the commissure should have been. These projections did not touch by at least onequarter of an inch. A complete study of the specimen does not seem to have been made.

A still more important case, and one more closely resembling mine, has been reported by Haab. ${ }^{2}$ His specimen was from a girl, twenty-seven years old, who had the development of a child of fourteen or fifteen years, and from her appearance had not reached puberty. She was an idiot, and had much difficulty in walking. The optic chiasm was entirely absent, but the optic tracts were present as small bands. The oculomotorius, abducens, and trochlearis appeared to be normal, and yet the merest trace of an eyeball was found. The muscles of the orbits appeared to be normal. The external geniculate body was entirely absent, but the internal geniculate body was of normal size. The corpora quadrigemina were of normal size macroscopically. The optic foramen was almost obliterated. The pulvinar of the optic thalamus was smaller than in a normal brain.

The conclusions that may be drawn from my case are:

- Transactions of the American Ophthalmological Society, 1885, p. 367.

2 Beiträge zur Ophtalmologie als F'estgabc. Friedrich Horner, 1881, p. 131. 
(1) The chief "primary" optic centre is the external geniculate body.

(2) The pulvinar of the optic thalamus is also an important " primary" optic centre.

(3) The anterior colliculus of the quadrigeminal body in man has an unimportant relation to vision.

(4) The subthalamic body, the habenula, the internal geniculate body, probably are not part of the visual system.

(5) The cortex of the calcarine fissure may contain nearly the normal number of cell bodies, even though the visual system may be undeveloped.

(6) The nerves to the ocular muscles and their nuclei may be developed, even though the visual system is absent.

(7) Congenital spastic paraplegia may be the result of deficient formation as regards number or size of the neurones of the central motor system, even though such a deficiency may be difficult to detect by the microscope.

\section{Fig. 1.}

A photograph of T. S., taken after death. The right upper limb, the fingers of both hands, and the lower limbs are slightly contractured. The palpebral fissures are small.

\section{Fig. 2.}

The occipital lobes have been cut away. A portion of the right temporal lobe has also been cut away in order to show the complete absence of the visual system. The internal geniculate body (A) is well shown, but no trace of the external geniculate body or of an optic tract or chiasm can be found.

\section{Fig. 3.}

The optic thalami are well shown. The anterior colliculi of the corpora quadrigemina, especially the right colliculus, appear smaller than the posterior, because the former were further from the camera.

Fig. 4.

The left ascending frontal convolution in the centre for the upper limb (B) is very narrow.

FIG. 5.

The upper part of the right cerebral hemisphere, showing the small cuneus (C). 
Fig. 6.

Photograph of a horizontal section of one cercbral hemisphere, showing Meynert's commissure (B) well developed, and also the internal geniculate body (C), while the external geniculate body is entirely absent.

\section{FIG. 7.}

A drawing of a horizontal section through the basal ganglia of one hemisphere. The fibres entering the posterior part of the optic thalamus are few in number.

\section{F'IG. 8.}

Drawings of transverse sections through portions of the abducent nerves. The upper drawing represents the fine fibres of the left abducent nerve, and the lower drawing the sclerotic areas in the right abducent nerve. 


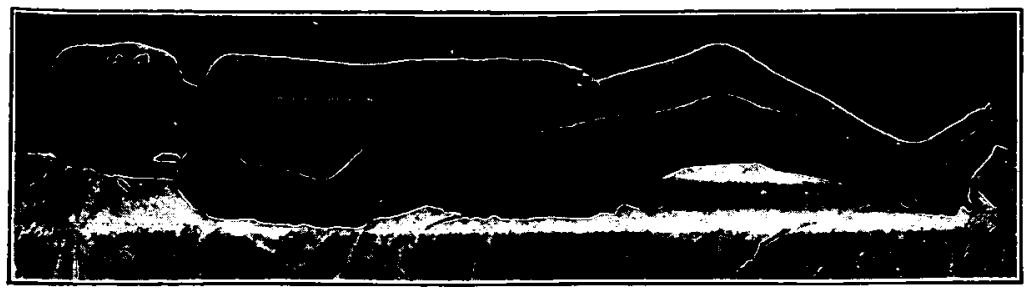

Erg. 1.

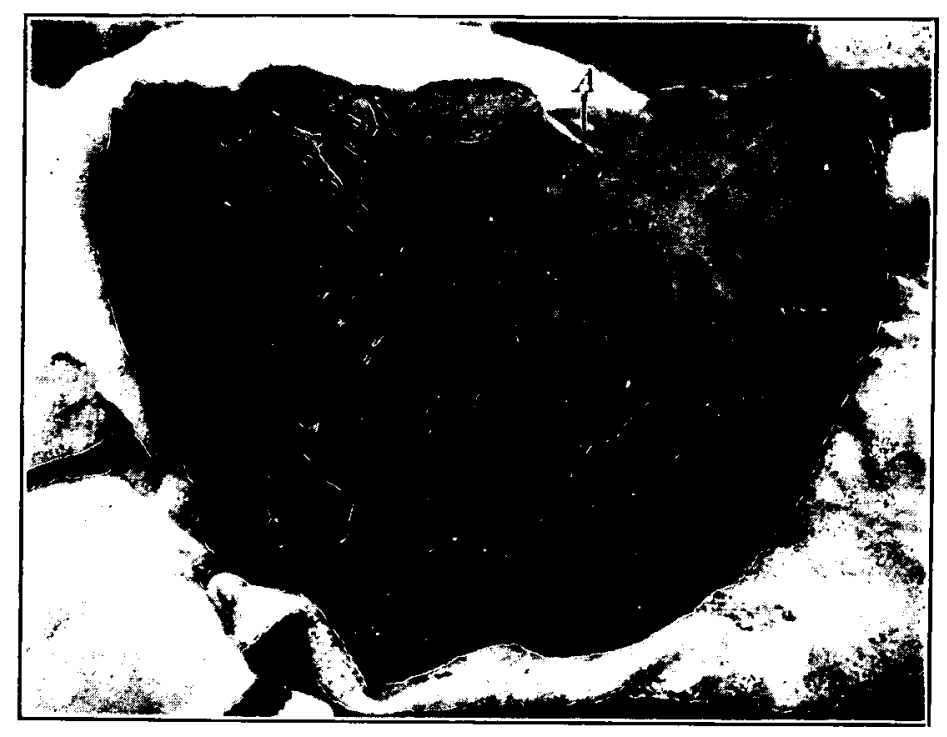

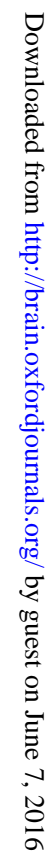

FIG. 2. 


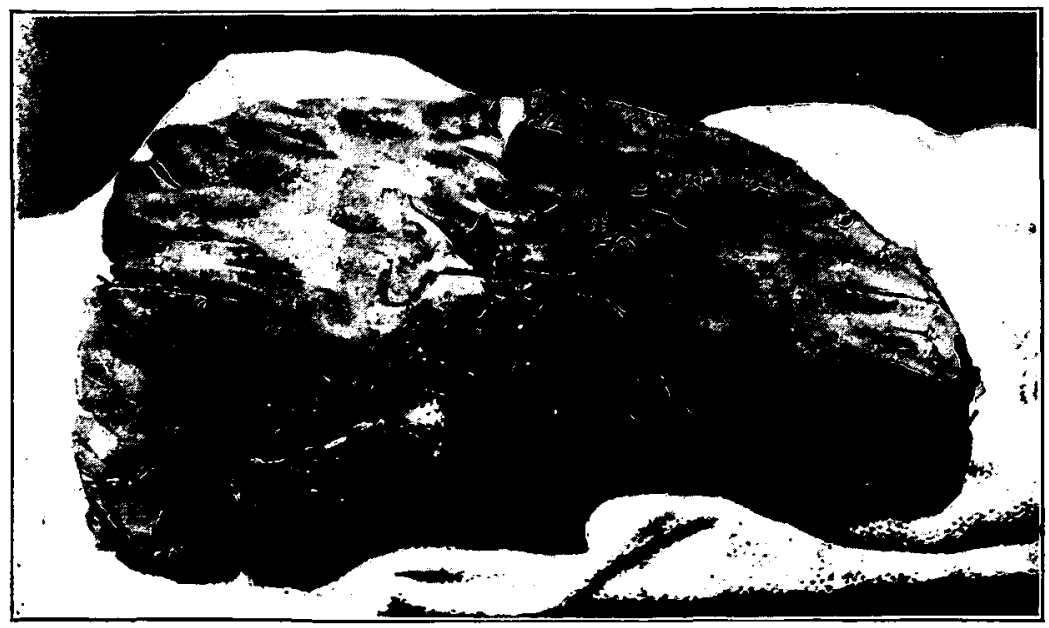

FIG. 3.

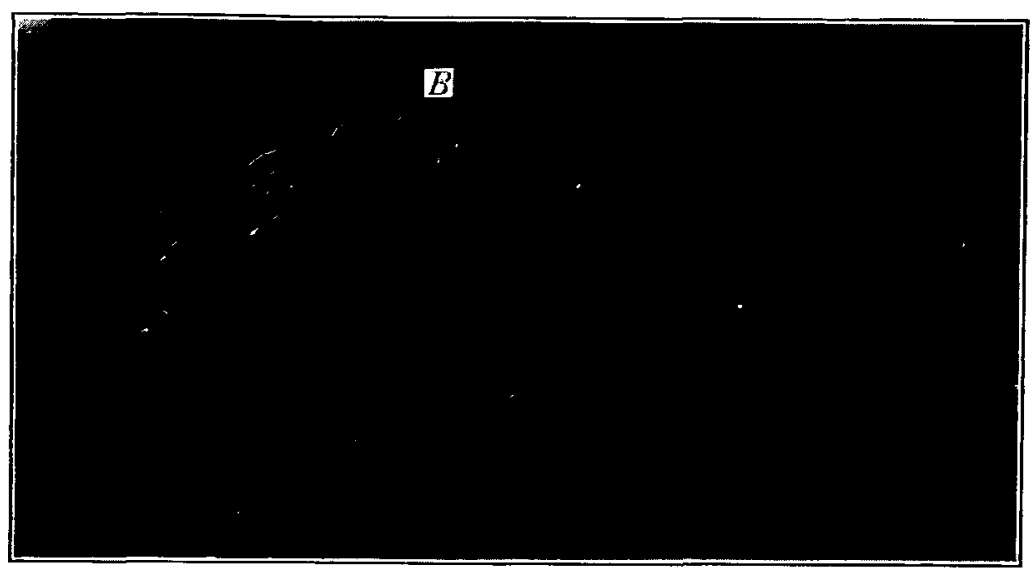

Fig. 4.

0
0
0
0
0
0
00
0
0
0
0
0
0
0
0
0
0
0
0
0
0
0
0
0
0
0
0
0
00
0
0
00
0
0
0
0
0
0
0
0
0
0
0
0
0

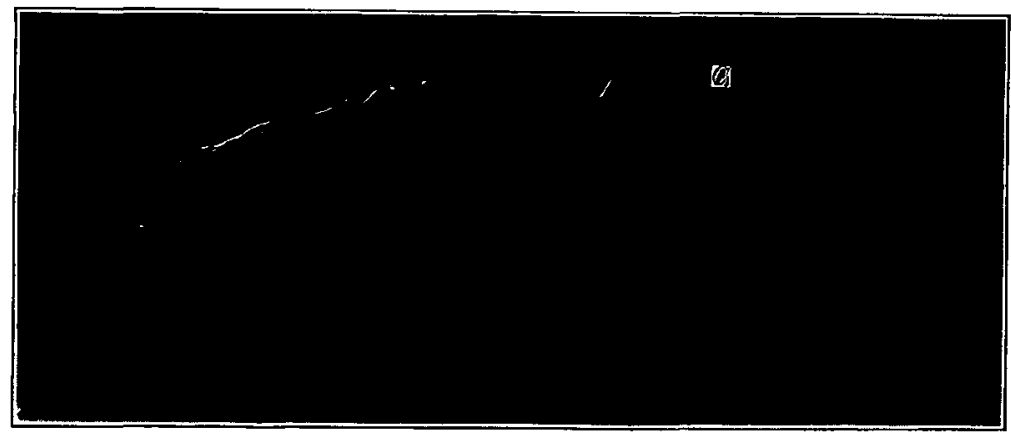

Fig. 5. 


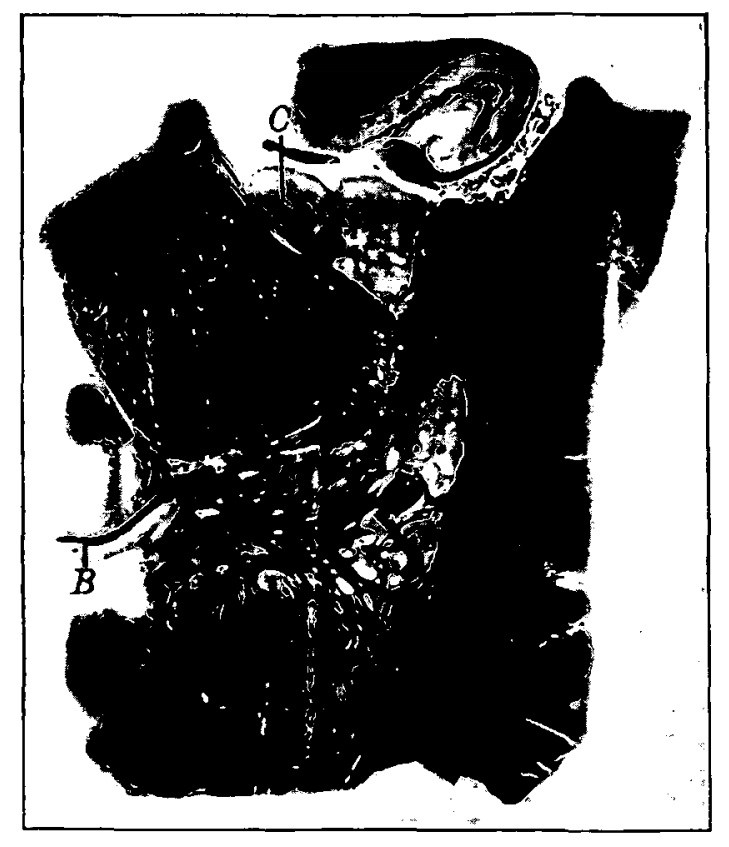

FIG. 6.

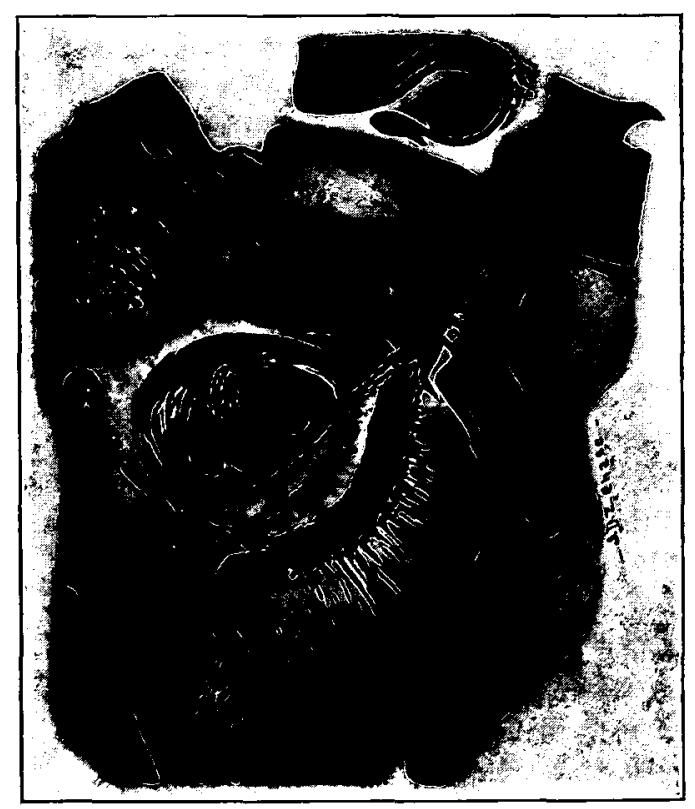

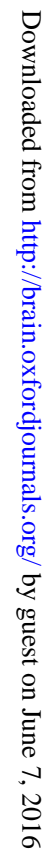

FIG. 7. 


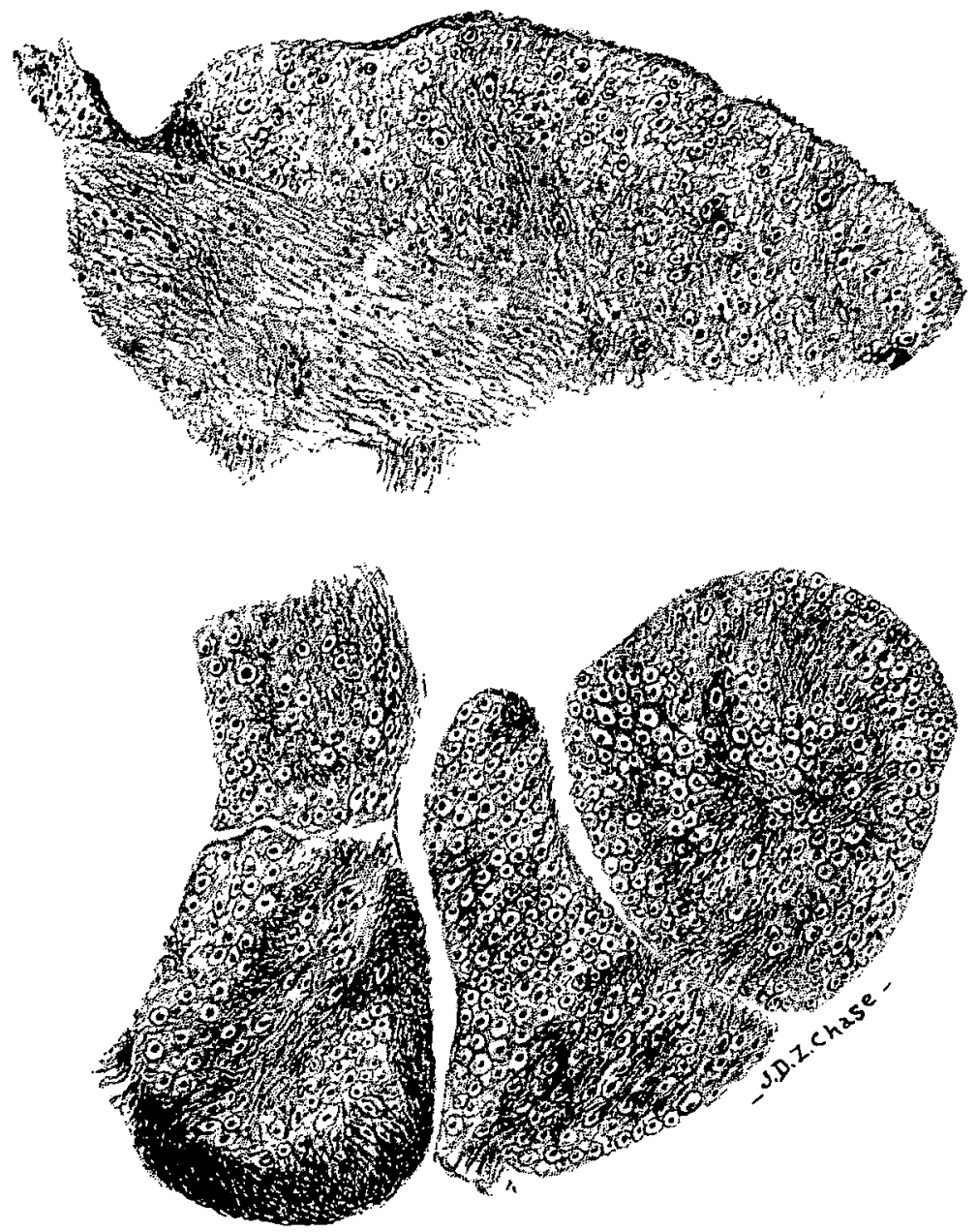

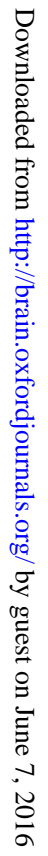

Fig. 8. 